\title{
Microstructure and Properties of 3D Ti-6Al-4V Articles Produced with Advanced Co-axial Electron Beam \& Wire Additive Manufacturing Technology
}

\author{
Dmytro Kovalchuk ${ }^{1}$, Orest Ivasishin ${ }^{2}$, Dmytro Savvakin ${ }^{2}$ \\ ${ }^{1}$ JSC NVO Chervona Hvilya, (Kyiv, Ukraine) \\ ${ }^{2}$ G. V. Kurdyumov Institute for Metal Physics (Kyiv, Ukraine)
}

Ti-6Al-4V articles were produced with advanced additive manufacturing technology of Direct Energy Deposition (DED) type using profile electron beam and wire as feedstock material. The key distinctive feature of this additive manufacturing process is the applying of the hollow conical electron beam generated by low-voltage $(<20 \mathrm{kV})$ gas-discharge EB gun for heating and melting of the substrate and co-axially fed wire. Such configuration ensures precisely controllable liquid metal transfer from the wire end to the substrate, specific temperature gradients at the fusion area and heat flow from liquid metal pool. Such conditions of heating, melting and cooling during 3D manufacturing processing provide the ability for controllable microstructure formation, including grain size and material texture.

Influence of processing parameters and cooling conditions on crystallization, grain formation and intragrain structure of solidified material is discussed. Optimization of processing parameters allowed production of $3 \mathrm{D}$ Ti-6Al- $4 \mathrm{~V}$ articles with isotropic microstructure and mechanical properties which met standard requirements for Ti-6Al-4V alloy.

\section{Introduction.}

Additive manufacturing (AM) of metals, which initially and for a long time has been considered as the technology for prototyping and research, is increasingly moving toward the category of real industrial technologies [1]. In recent years, this trend has begun to spread to a wider range of industries, including aerospace, and to products of quite a large size $[2,3]$.

This trend has special requirements, not only in terms of external perfection and geometric accuracy, but also with respect to the quality of materials formed by layer-upon-layer deposition or sintering. The most important are the mechanical properties, which are provided by various complex factors, such as the chemical composition of 3D printed material, the appearance of defects of various nature, metal structure, residual stresses, etc., which, in turn, are the result of the interaction of complex parameters of a particular printing process.

Many existing metal AM methods have already been thoroughly investigated, resulting in already defined opinion about the potential of a particular technology, the maximum achievable properties, the complexity and sometimes the impossibility of preventing some technological problems. The most critical problems of many metal AM technologies are residual defects (pores, lack of fusions, voids), residual stresses and deformations caused by large temperature gradients in the manufactured product, non-uniform properties in different directions, which are particularly associated with the formation of a columnar metal structure in the direction of product growth $[4,5,6]$.

The main efforts of developers of AM metal technologies and suppliers of 3D printing equipment are namely aimed at solving existing technological problems and constraints, primarily by improving control over a complex set of process parameters.

Furthermore, the metal AM market is looking for new technical solutions that can solve these problems by applying new innovative approaches to providing enhanced physical and metallurgical conditions for the formation of deposited or sintered material.

The new additive manufacturing technology of Direct Energy Deposition (DED) type which employs the unique profile electron beam and wire as feedstock material has been invented and patented [7] in Ukraine to solve most of existing problems of metal AM. This technology was called the xBeam 3D Metal Printing thanks to the character view of the electron beam. It provides the combination of exclusive technical and technological features which allow achievement of the sufficient quality of produced 3D-printed metals with high productivity and good accuracy.

There are key physical and engineering ideas that provide the main technological capabilities to xBeam 3D Metal Printing: 1) hollow conical electron beam is used for creation of molten pool on the substrate and for melting of feedstock material; 2) feedstock is supplied exactly in the center of molten pool through feedstock guide coaxially with electron beam; 3) low voltage gas discharge EB gun (xGun) and feedstock guide are forming together a common functional assembly (see general design and process configuration of xBeam on Fig.1 and Fig.2 accordingly). 


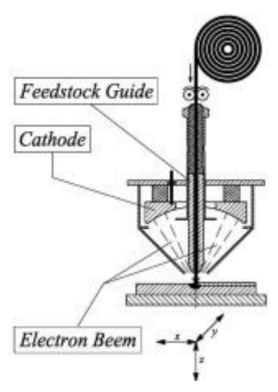

\section{Fig.1. General design of the xBeam}

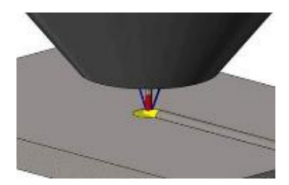

Fig.2. Process configuration of the xBeam

The round molten pool and vertical supply of feedstock exactly in the center of the pool provide the best power efficiency of the process. Such configuration excludes any shadow zones on the substrate preventing porosity and other macro defects like lack of fusion and cavities in deposited layer. The hollow conical electron beam completely envelopes the feedstock material by precisely regulating the energy flow (Fig.3). It provides absolutely uniform pre-heating and fusing of wire feedstock resulting in continuous transfer of the molten metal from the wire end to the molten pool on the substrate. This flow of molten metal is reliably maintained by the "bridge" of surface tension forces. Molten metal is uniformly spread around the deposition point within limits of the molten pool to form smooth and accurate beads on the substrate along the movement of the deposition point (Fig.4).

Such an axisymmetric configuration of thermal fields and mass transfer greatly simplifies the mathematical modeling of the process, which is very important for improving the control of the technological process in order to obtain the predicted and specified material properties [8]. 
Fig.3. Hollow conical electron beam and its effect on the wire

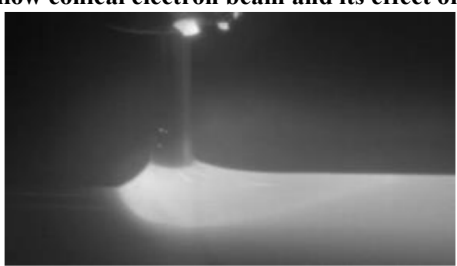

Fig.4. Liquid metal transfer from the wire end to the substrate

The parameters of the deposited bead (width and thickness) are easily controlled by changing the power distribution between the wire and substrate by regulation of the protrusion between the xGun and the substrate, also by regulation of the substrate speed and the wire feed rate (Fig.5). Different parameters of the deposited beads result in a different cooling rate and solidification conditions, providing control of the received metal structure. Thus, a reliable and at the same time flexible mechanism for forming a deposition bead of a predetermined geometrical parameters and basic metalstructure of the 3D part to be produced is realized. Any required thickness of the 3D-printed part can be provided by using a deposition strategy consisting of a few parallel beads with some overlap between adjacent beads (Fig.6).

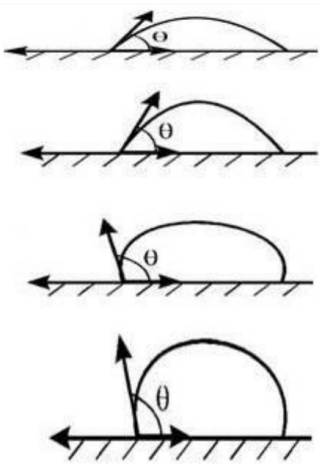

Fig.5. Different configurations of the deposited beads depending on deposition conditions

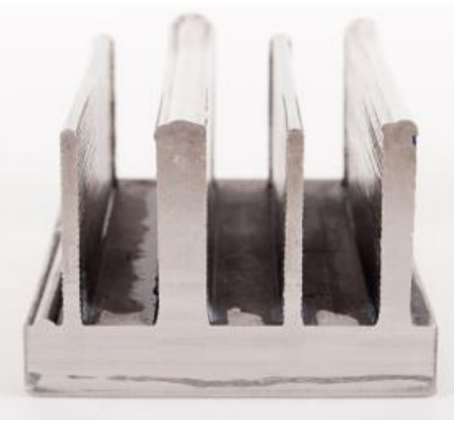

Fig.6. Walls of different thickness $-4,8$ and $12 \mathrm{~mm}$

Beads with very different parameters were obtained using this approach - from a layer thickness of $0.6 \mathrm{~mm}$ with a bead width of $4.2 \mathrm{~mm}$ (manufactured by wire Ø2mm) up to a layer thickness of $1.75 \mathrm{~mm}$ with a bead width of $3.3 \mathrm{~mm}$ (manufactured by wire $\varnothing 3 \mathrm{~mm}$ ) - see Fig.7a and $7 \mathrm{~b}$. In all cases very good accuracy and low roughness of the surfaces of the sidewalls was demonstrated, independent from the height and thickness of the samples (Fig.6). Usually the roughness did not exceed $0.5 \mathrm{~mm}$ (Fig.8) which results in a highly reduced amount of 
material and time losses for post- processing machining. In addition, there were no any surface defects such as cracks or voids both on the external surface and in the near-surface areas of the walls.

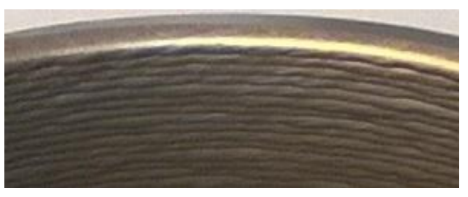

Fig.7a. Wall with the bead's thickness $0.6 \mathrm{~mm}$ and width $4.2 \mathrm{~mm}$.

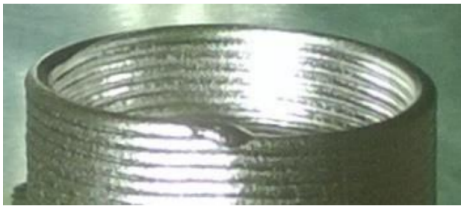

Fig.7b. Wall with the bead's thickness $1.75 \mathrm{~mm}$ and width $3.3 \mathrm{~mm}$

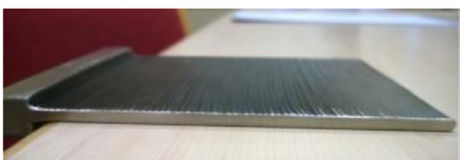

Fig.8. Wall with the average thickness $3.2 \mathrm{~mm}$ and maximum deviation $0.3 \mathrm{~mm}$ from the minimum value

An extremely important feature of the xBeam 3D Metal Printing technology is the significantly lower power concentration of the heating source (electron beam) generated by the low-voltage gas-discharge EB gun in comparison with any alternative heating sources being used in other currently known metal additive manufacturing processes. The special gas-discharge EB gun designed for the xBeam 3D Metal Printing technology (xGun) generates an electron beam at a low accelerating voltage less than $20 \mathrm{kV}$. The ultimate power level of the electron beam is approximately $20 \mathrm{~kW}$ and it has a minimum focus of approximately $2.0 \mathrm{~mm}$ in diameter and provides very soft and smooth heating of processed surfaces without scanning. The power concentration in the focus of this electron beam does not exceed $10^{3} \mathrm{~kW} / \mathrm{cm}^{2}$.

The impact of such a beam on the surface makes it possible to form a very shallow molten pool, which is sufficient to create conditions for spreading the incoming liquid metal above the maintained liquid phase on the substrate with minimal heat affected zone on the substrate, around and below the deposition area.

Such low specific power input with gradual reduction of power heating intensity from the maximum to the periphery highly reduces residual stresses and distortions of the produced parts, which currently is considered as one of the critical problems of existing metal AM methods [4].

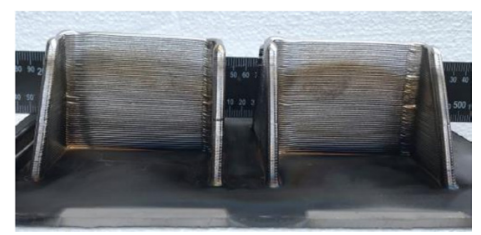

Fig.9. Sample made by depposition of Ti-6Al-4V wire $3 \mathrm{~mm}$, substrate plate Ti-6Al-4V thickness $5.0 \mathrm{~mm}$

The creation of a rough column structure in deposited materials is one of the serious problems of existing metal AM technologies. Such type of structure results in non-uniform properties of the deposited material in different directions, especially it influences the plasticity of the 
formed material. This problem sometimes is so critical that additional thermo-mechanical processing such as rolling or forging is applied in order to break these columns and to form a fine and uniform metal structure similar to wrought material [9, 10]. These steps increase the general operating costs and lead time, resulting in the general reduction of additive manufacturing attractiveness.

As result of this painful problem even the new developed standards on material produced by 3D-printing permit different properties in the direction of growth and along the deposited layers, and “...columnar grain structure in the deposit is acceptable..." [11, 12].

The purpose of the further discussed experiments was to demonstrate the ability to form an acceptable metal structure directly by $3 \mathrm{D}$ printing, and to provide good metal properties which meet the requirements of existing standards on appropriate materials.

\section{Experiments}

An exploratory study of the technological capabilities of xBeam 3D Metal Printing was executed by conducting experiments with the titanium alloy Ti-6Al-4V. This grade is the most popular titanium alloy in different industries owing to its excellent combination of high mechanical and technological properties. Good fatigue strength, which can be achieved in this alloy by using a complex of different technological processes, caused this alloy to become one of the most important structural materials in aerospace industry. Because of this fact the main efforts of scientists and engineers in the field of metal AM involved an investigation of the properties of Ti- $6 \mathrm{Al}-4 \mathrm{~V}$ provided by metal 3D printing.

Mechanical properties of all materials, including titanium-based alloys produced with additive manufacturing technologies, depend on their microstructure. The higher is content of alloying elements in titanium alloys, the wider range for controllable variation of their mechanical parameters is possible owing to microstructure changes. Thus, $\alpha+\beta$ Ti-6Al-4V material demonstrates wider opportunities for variation of mechanical characteristics as compared to near- alpha titanium compositions but has less potential than near-beta and beta titanium compositions. To achieve appropriate set of mechanical characteristics size and aspect ratio (shape) of $\beta$ grains as well as their inner microstructure should be controlled. For lamellar $\alpha+\beta$ microstructure formed by used AM processing, size of $\beta$ grains, size of $\alpha$ colonies inside them and thickness of $\alpha$ lamellae are critical parameters affecting mechanical properties. The $\alpha$ colony size determines effective length of dislocation slip through entire colony [13], thus lower size of $\alpha$ colonies with similar orientation of lamellae provides higher ductile and strength characteristics as well as better high cycle fatigue properties. Small $\beta$ grains are preferable because of grain size restricts $\alpha$ colony sizes. Moreover, at large $\beta$ grains, long grain boundary $\alpha$ phase layers are weak microstructure elements responsible for premature fracture due to strain difference between intragrain structure and the grain boundary. Presence of elongated $\beta$ grains which aspect ratio is quite bigger than 1 results in non-isotropic mechanical characteristics of the material and, generally, is harmful for mechanical characteristics. As for intragrain $\alpha$ phase, its morphology and size determines mainly strength level and fatigue characteristics and strongly depends on heat treatment parameters. During additive manufacturing process, formation of $\beta$ grains during melt crystallization and formation of intragrain $\alpha+\beta$ structure upon further cooling below

$\beta$-transus temperature are determined by cooling rate, which in turn, considerably depends on heat flow and temperature gradients formed around heated area. It is very important to understand that temperature gradients in the process of layer-upon-layer deposition depends not only on main process parameters like power, feed rate and substrate speed but also on the heat accumulated in previously deposited material along the whole process duration. That is the thermal history must be considered as one of the most important factors of formation of metal structure.

So, to attain appropriate mechanical characteristics of Ti-6Al-4V material produced with AM technology, the aim was to produce uniform relatively fine-grained microstructure without harmful elements like elongated grains with long grain boundary $\alpha$ layers.

\section{$\underline{\text { Results and Discussion. }}$}

The metal structure and properties obtained by layer-by-layer deposition by xBeam 3D Metal printing were investigated on the samples formed by single-bead and triple-bead strategy (by three parallel passes).

The macrostructure of the single-bead sample in the Z-Y plane is shown in Fig.10a (middle zone) and Fig.10b (top zone) accordingly. The macrostructure of the single-bead sample is mostly formed by grains sized 200-600 $\mu$ m, which grow through the border between the nearest beads. Grains near the surface are slightly elongated in the direction from the center to surface under some angle up to $45-50^{\circ}$ from the vertical direction, whereas grains in the center are practically equiaxed. 


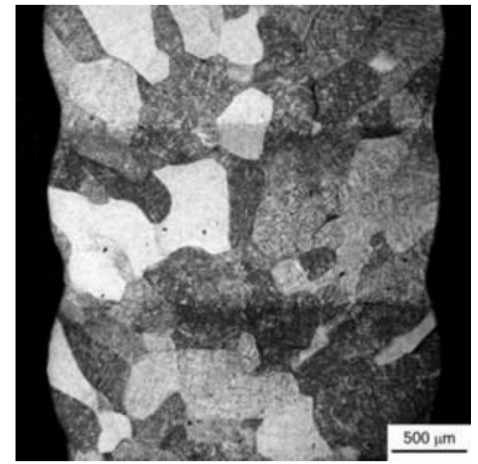

Fig.10a. Macrostructure of the single-bead sample in Z-Y plane_middle zone

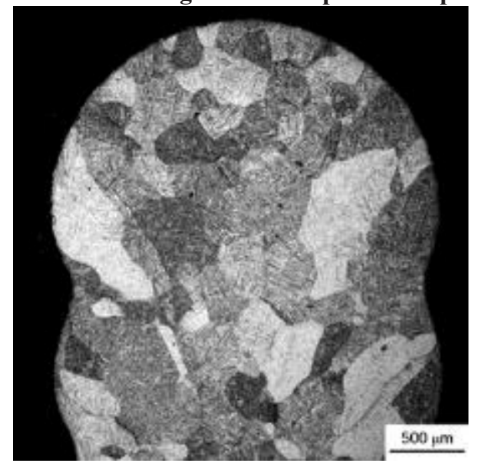

Fig.10b. Macrostructure of the single-bead sample in Z-Y plane, top zone

The macrostructure of the triple-bead sample in the Z-Y plane is shown in Fig.11.

Microstructure investigations revealed that $\beta$ grains with average size of $400-\mu \mathrm{m}$ were formed upon melt crystallization during AM processing. Intragrain microstructure consists mostly of fine parallel $\alpha$ lamellae which were formed on cooling, however, martensite type microstructure was also observed in some grains. Thus, it can be concluded that average cooling rates in our experiments were close to critical value above of which martensite transformation was realized while diffusion type $\beta \rightarrow \beta+\alpha$ phase transformation was also occur at a bit slower cooling.

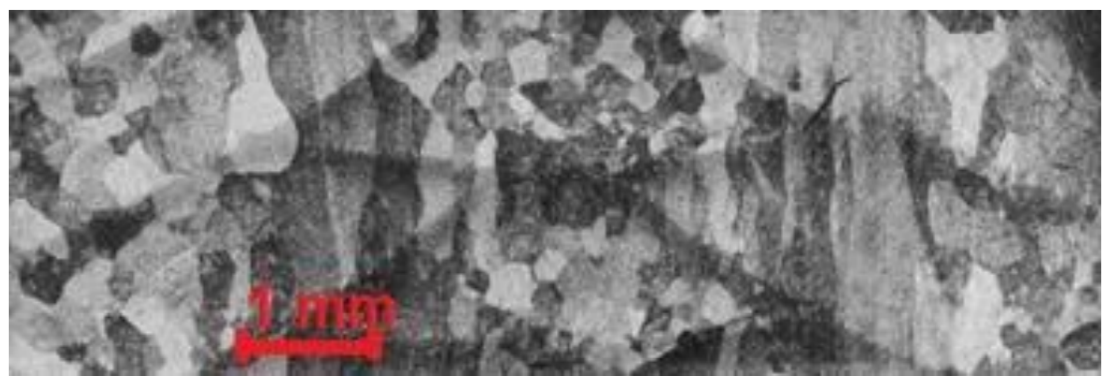

Fig.11. Microstructure of the triple-bead sample in Z-Y plane 
Detailed investigation of grain sizes shown that grains formed at the first level adjacent to cold substrate (Fig.12a) are noticeably smaller (of about $300 \mu \mathrm{m}$ ) than grains in the core of AM structure (Fig.12b). It can be explained by intensive heat flow towards cold substrate and significantly faster cooling of the material at the first crystallized layer. Similar grains some smaller than average grain size over AM produced structures were also formed at upper crystallized layer (Fig.12c) due to intensive heat flow via irradiation and faster cooling of such layers. It was concluded, some additional contribution to grain growth inside intermediate layers of produced material gave heat flow during subsequent melting of neighboring upper layers.

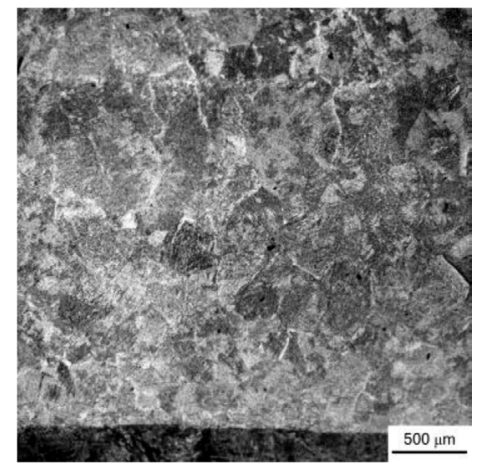

Fig.12a. Macrostructure of the multi- bead sample in Z-Y plane, interface with substrate

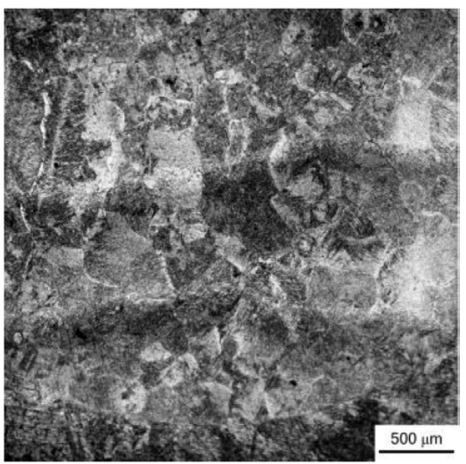

Fig.12b. Macrostructure of the multi-bead sample in Z-Y plane, middle zone

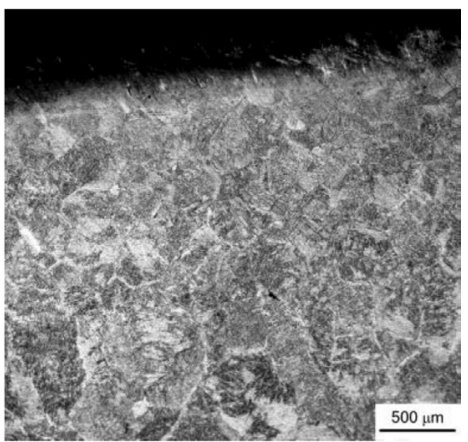


Fig.12c. Macrostructure of the mulit-bead sample in Z-Y plane, top zone

Under the some heating and cooling regimes applied for 3D manufacturing processing, large columnar $\beta$ grains elongated in vertical directions and extended throughout the number of layers were observed at the boundaries between two former molted beads (Fig.11). It was suggested that heat flows and temperature gradients formed around molted beads as well heat affecting zones arose during building of next (upper) layers resulted in continuous crystallization growth of grains at the borders between two neighbor beads.

In spite of the differences in some of the characteristics of grains in different zones of the sample cross-section in the Z-Y plane, measuring the microhardness has demonstrated a very uniform picture of the Vickers hardness mapping in all zones excluding the area near the substrate (Fig.13).

A consistent and practically uniform metal structure, together with a good chemical composition of the material produced by xBeam 3D Metal Printing, results in good mechanical properties. The set of single-bead and triple-bead samples of titanium alloy Ti-6Al-4V was produced on the pilot installation for basic mechanical testing (tensile strength, yield strength, elongation, and reduction of the area). Specimens were cut from each sample both in the direction of deposition of the layers (in the horizontal direction X) and across the layers (in the vertical direction Z).

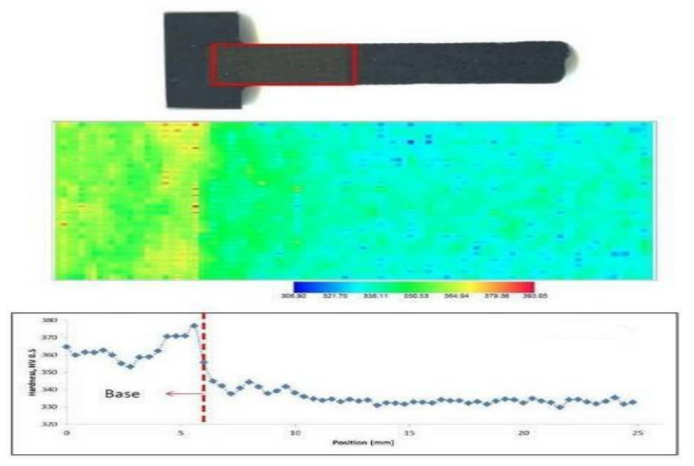

Fig.13. Microhardness of the triple-bead sample in Z-Y plane

The testing results presented in Table 1 demonstrate that all specimens comply with the requirements of the main standards.

Table 1. Mechanical properties of single-bead and triple-bead samples of titanium alloy Ti-6Al-4V deposited by xBeam 3D Metal Printing compared with industrial standards

\begin{tabular}{|c|c|c|c|c|c|c|}
\hline Specification & \multicolumn{2}{|c|}{ Tensile strength, MPA (ksi) } & \multicolumn{2}{|c|}{$0.2 \%$ Yield strength, $\mathrm{MPa}(\mathrm{ksi})$} & \multicolumn{2}{|c|}{ Elongation, $\%$} \\
\hline & $(\mathrm{X}-\mathrm{Y})$ & $(\mathrm{Z})$ & $(\mathrm{X}-\mathrm{Y})$ & (Z) & $(\mathrm{X}-\mathrm{Y})$ & $(\mathrm{Z})$ \\
\hline AMS 4999, min & $\begin{array}{c}889 \\
(129)\end{array}$ & $\begin{array}{c}855 \\
(124)\end{array}$ & $\begin{array}{c}800 \\
(116)\end{array}$ & $\begin{array}{c}765 \\
(111)\end{array}$ & 6 & 5 \\
\hline AMS 4928 (Forging Annealed) & $\begin{array}{c}896 \\
(130)\end{array}$ & & $\begin{array}{c}827 \\
(120)\end{array}$ & & 10 & \\
\hline AMS 4905 (Beta Annealed) & $\begin{array}{c}841 \\
(122)\end{array}$ & & $\begin{array}{c}745 \\
(108)\end{array}$ & & & \\
\hline $\begin{array}{l}\text { xBeam 3D Metal Printing } \\
\text { (triple bead samples) }\end{array}$ & $\begin{array}{c}940-949 \\
(136-138)\end{array}$ & $\begin{array}{c}913-926 \\
(132-134)\end{array}$ & $\begin{array}{c}848-873 \\
(123-127)\end{array}$ & $\begin{array}{c}829-842 \\
(120-122)\end{array}$ & $12-17$ & $12-17$ \\
\hline $\begin{array}{l}\text { xBeam 3D Metal Printing } \\
\text { (single bead samples) }\end{array}$ & $\begin{array}{c}906-917 \\
(131-133)\end{array}$ & $\begin{array}{c}907-934 \\
(132-133)\end{array}$ & $\begin{array}{c}825-837 \\
(120-121)\end{array}$ & $\begin{array}{c}827-850 \\
(120-123)\end{array}$ & $14-15$ & $13-14$ \\
\hline
\end{tabular}

Results of these investigations demonstrate practically equal values of strengths and elongation both in $\mathrm{X}$ and $\mathrm{Z}$ directions in single-bead samples, and some higher strength in $\mathrm{X}$ direction in triple-bead samples. This is in full correspondence with the practically isotropic character of macrostructure of single-bead samples and with macrostructure of triple-bead samples which content some colonies of grains elongated in $\mathrm{Z}$ direction.

One of the specimens was used to investigate the fracture surface after tensile testing. The actual characteristics of this specimen were the 
following: YS $=864 \mathrm{MPa}$, UTS $=947 \mathrm{MPa}, \mathrm{El} .=17 \%$, RA $=36 \%$. Images at different magnifications of the fracture surface of this specimen are shown in Fig. 14a, 14b, and 14c. The ductile type of fracture is observed, while brittle chips are not detected. Areas of a ductile fracture with small equiaxed pits of different sizes of $1-10 \mu \mathrm{m}$ were monitored over the entire facet area.

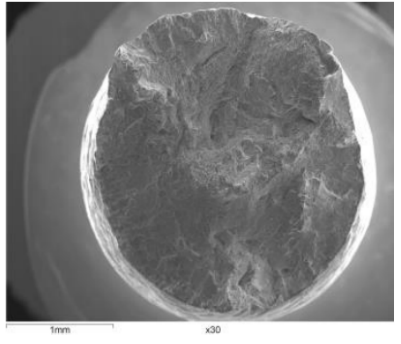

Fig.14a. Fracture surface of the specimen after tensile testing_x30

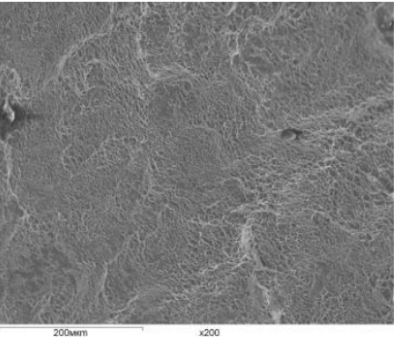

Fig.14b. Fracture surface of the specimen after tensile testing $\times 200$

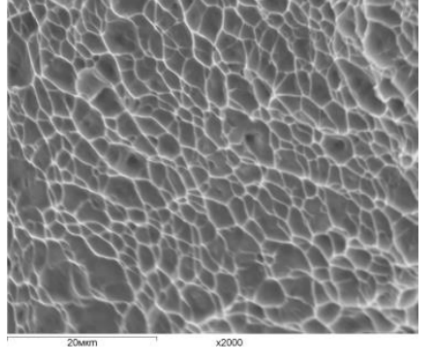

Fig.14c. Fracture surface of the specimen after tensile testing_x2000

Results of investigation of mechanical properties have demonstrated that columnar-like grains are harmful for achievement of isotropic mechanical properties, thus AM manufacturing regimes must be corrected to achieve uniform grain structure under melting/crystallization and followed heat affecting during melting/crystallization of adjacent upper layers.

Appropriate experiments were already performed with application of improved deposition strategy for building a multi-bead samples from CP Titanium and alloy Ti-6Al-4V. In these experiments each next layer was deposited with the overlap to the previous layer equal to 0.5 of bead width (see Fig.15). Obtained results have demonstrated practically isotropic character of macrostructure for both materials (see Fig.16a and Fig.16b). Investigations of mechanical properties of these materials 3D-printed using improved deposition strategy are in progress.

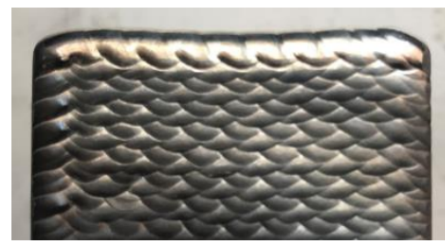

Fig.15. Deposition strategy with overlapping layers

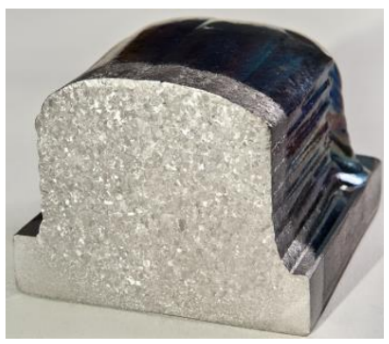

Fig.16a. The multi-bead CP Ti sample with isotropic macrostructure

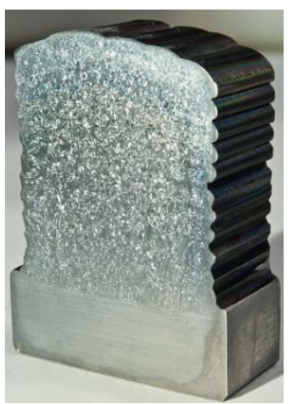

Fig.16b The multi-bead Ti-6Al-4V sample with isotropic macrostructure

\section{Conclusions}

- xBeam 3D Metal Printing has proved capable of providing an isotropic metal structure in deposited materials including CP Titanium and Ti-6Al-4V;

- Basic mechanical properties of Ti-6Al-4V produced by xBeam 3D Metal Printing meet the requirements of actual industrial standards;

- Manufacturing of accurate defect-free parts with high rate and good properties by xBeam 3D Metal Printing is able to provide an excellent buy-to-fly ratio which is very important for basic aerospace material such as Ti- $6 \mathrm{Al}-4 \mathrm{~V}$ and other expensive materials including titanium-based alloys, nickel-based alloys, niobium-based alloys, stainless steel, special steel grades, as well.

- Further investigations are required to comply with the main certification requirements.

The xBeam 3D Metal Printing technology and 3D metal printers of the xBeam family promise to become reliable, efficient, and friendly equipment for the widest industrial and scientific circles. 


\section{$\underline{\text { References }}$}

[1] Wohlers Report 2018. 3D Printing and Additive Manufacturing State of the Industry Annual Worldwide Progress Report. ISBN ISBN 978-0-9913332-4-0.

[2] Norsk Titanium to Deliver the World's First FAA-Approved, 3D-Printed, Structural Titanium Components to Boeing. - BusinessWire https://www.businesswire.com/news/home/20170410005330/en/Norsk-Titanium-Deliver-

World\%E2\%80\%99s-FAA-Approved-3D-Printed-Structural

[3] Lockheed Martin 3D Prints Large Titanium Domes for Satellite Fuel Tanks. - Sarah Saunders. - Jul 12, 2018, 3D Printing, Aerospace 3D Printing, Metal 3D Printing. - https://3dprint.com/219196/lockheed-martin-fuel-tank-domes/

[4] Marc Saunders. Boosting AM adoption - the next phase of market growth. - May 29, 2018. - https://www.linkedin.com/pulse/boostingam-adoption-next-phase-market-growth-marc-

lipi=urn\%3Ali\%3Apage\%3Ad_flagship3_feed\%3BWi308Uv1SqusaSo4Gsyydw\%3D\%3D.

[5] Cristian Decaillet. Additive Manufacturing - Challenges to Face. - TITANIUM 2017 Conference, October 8-11, 2017, Florida, USA.

[6] Steffen Beyer. Industrialization of Additive Manufacturing for Space Parts - Hurdles and Challenges. - International Symposium of Additive Manufacturing, February 25-26, Dresden, Germany.

[7] Patent of Ukraine No.112682 «Method and Apparatus for manufacturing of three dimensional objects», 2017

[8] O.V. Makhnenko, A.S. Milenin, E.A. Velikoivanenko, N.I. Pivtorak and D.V. Kovalchuk. Modelling of temperature fields and stressstrain state of small 3D sample in its layer-by-layer forming. The Paton Welding Journal, 2017, 3, p. 714.

[9] Paul Colegrove, Stewart Williams. - High deposition rate high quality metal additive manufacture using wire + arc technology. Presentation at Cranfield University, UK.

[10] Arconic Offers Hybrid Ampliforge Process for High-Performance Aerospace Components. - Additive Manufacturing Magazine. May 4, 2018. - https://www.additivemanufacturing.media/products/arconic-offers-hybrid- ampliforge-process-for-high-performance-aerospacecomponents.

[11] AMS 4999.

[12] AMS 7004.

[13] G. Lutjering, J. Williams. Titanium, 2nd ed., 2007. 